\title{
ILCEA
}

Revue de l'Institut des langues et cultures

d'Europe, Amérique, Afrique, Asie et Australie

43 | 2021

Images des Amériques : fabrique, représentations, usages

\section{Le Pérou et ses habitants : les films-portraits de Lionel Terray (1921-1965) et leur contexte de production}

Peru and Its Inhabitants: The Lionel Terray's Portraits-Films (1921-1965)

\section{Chloé Tessier-Brusetti}

\section{OpenEdition}

\section{Journals}

Édition électronique

URL : https://journals.openedition.org/ilcea/13428

DOI : $10.4000 /$ ilcea. 13428

ISSN : 2101-0609

Éditeur

UGA Éditions/Université Grenoble Alpes

Édition imprimée

ISBN : 978-2-37747-299-4

ISSN : $1639-6073$

Référence électronique

Chloé Tessier-Brusetti, « Le Pérou et ses habitants : les films-portraits de Lionel Terray (1921-1965) et leur contexte de production», ILCEA [En ligne], 43 | 2021, mis en ligne le 30 juin 2021, consulté le 01 juillet 2021. URL : http://journals.openedition.org/ilcea/13428 ; DOI : https://doi.org/10.4000/ilcea. 13428

Ce document a été généré automatiquement le 1 juillet 2021

(C) ILCEA 


\section{Le Pérou et ses habitants : les films- portraits de Lionel Terray (1921-1965) et leur contexte de production}

Peru and Its Inhabitants: The Lionel Terray's Portraits-Films (1921-1965)

Chloé Tessier-Brusetti

\section{Lionel Terray, de l'alpinisme à la pellicule}

1 Lionel Terray naît à Grenoble en 1921 dans une famille de "bourgeois aisés », selon ses propres termes ${ }^{1}$. Son père, après des études d'ingénieur chimiste, part fonder une industrie au Brésil ; mais la Seconde Guerre mondiale l'obligera à rentrer rapidement en France, avec sa femme, pour s'acquitter de son devoir de soldat. Dans son autobiographie (Terray, 1961), il reconstruit l'image de sa famille : un père passionné de compétitions automobiles, qui reprend des études en médecine après 40 ans d'affaires, une mère audacieuse qui skie en pantalon et conduit sa propre voiture à une époque où les femmes commencent tout juste à s'émanciper, des aïeux "grands voyageurs en quête de fortune et d'aventure ». Terray, lui, se considère avant tout comme un montagnard, tel que la littérature a figé ce personnage dans les esprits: « un rude paysan aux manières frustes » confie-t-il; il se décrit d'ailleurs de nature « réservée et timide » (Terray, $1961: 3-4)$.

2 Pendant longtemps, il a imaginé que la photographie était "une cuisine magique » difficile à maîtriser (ibid. : 425) et que les photographes-cinéastes qui accompagnent les expéditions ne servent pas à autre chose qu'à ternir son plaisir d'alpiniste. Mais en 1952, pendant sa première expédition dans les Andes, à l'occasion de l'ascension de l'Aconcagua, il découvre le plaisir de fixer des images sur la pellicule lorsque son ami Georges Strouvé lui prête un appareil photo et une caméra. Emballé par les résultats de ses clichés et rushes qu'il jugera plutôt convenables, il voit dans ces images de 
formidables souvenirs des courses auxquelles il a participé, mais également des documents qu'il pourrait utiliser pour collecter des fonds et financer de nouvelles expéditions. En 1950, l'ascension du premier sommet de plus de 8000 mètres, l'Annapurna, avait rapporté une belle popularité et fortes royalties à Maurice Herzog qui avait centré sur lui toute la gloire de cette conquête. Ses compagnons d'expéditions, dont faisaient partie Marcel Ichac, Lionel Terray et Louis Lachenal (entre autres), avaient signé un contrat d'exclusivité les contraignant à rester dans l'ombre de l'histoire officielle et à l'écart des bénéfices économiques engendrés par ces exploits sportifs. Terray se lance donc, dès 1952, dans la réalisation d'un premier film sur la descente à ski de la face nord du mont Blanc, dont il est le protagoniste, puis tourne quelques images sur la vie et les mœurs des populations de l'Himalaya lors de son ascension de l'Annapurna en 1955. L'exploitation de ces deux premiers films lui permettra de financer sa seconde expédition dans les Andes en 1956, longue de six mois, mais qu'il décidera de prolonger, estimant qu'il est «stupide de se rendre si loin pour une période si courte » (ibid. : 442). Deux mois de plus qu'il mettra à profit pour gravir deux cimes de plus de 8000 mètres et tourner des images sur la vie des Indiens quechuas. Il rapportera de nouvelles bobines sur les Indiens des montagnes péruviennes en 1962, lors de la première de la face est du Chacraraju. Ces dernières bandes viendront compléter les images des premiers rushes pour constituer le film C'est le Pérou, monté par ses amis cinéastes, Jean-Jacques Languepin et Marcel Ichac ${ }^{2}$, le spécialiste de films de montagne et de l'exploration française d'alors. Trois ans plus tard, Terray meurt après une terrible chute lors d'une ascension dans le Vercors en compagnie de son camarade Marc Martinetti.

\section{Images rapportées du Pérou}

3 Une partie des images tournées au Pérou par Lionel Terray et conservées par la famille a été numérisée, archivée et mise en ligne par la Cinémathèque d'images de Montagne (CIMALPES) : plusieurs heures de bobine, à la fois des rushes, des films montés et des chutes d'images. Les lieux fixés sur la pellicule sont ceux que Terray a visités : la région cusquénienne, l'Altiplano et le littoral.

Dans l'ensemble des images qu'il rapporte du Pérou, on peut identifier trois périodes de tournage, qui correspondent à ses trois expéditions dans les Andes (1952, 1956 et 1964), et huit films d'une durée variant entre 15 et 25 minutes approximativement. La moitié traite des expéditions et des ascensions, l'autre moitié dépeint les autochtones dans leur vie quotidienne ${ }^{3}$. Le dernier film (C'est le Pérou) est un montage réalisé à partir de ses différents documents sur les Indiens.

5 Trois grandes thématiques se détachent de ce corpus d'images: d'abord, la nature andine, avec ses vastes paysages et la démesure de la Cordillère; ensuite, les occupations quotidiennes des habitants, comme les tâches agricoles (l'élevage de camélidés, les récoltes) ou les travaux d'artisanat (la confection des vêtements traditionnels); enfin, les fêtes et les cérémonies rituelles (danses costumées, processions catholiques, célébration de l'Inti Raymi). Il s'attarde d'ailleurs largement sur cette dernière thématique et filme notamment la fin des fêtes où l'ébriété des gens est manifeste. On note que Terray cherche à filmer la vie de ces gens telle qu'elle s'offre à lui, dans sa différence et son authenticité; les images qu'il montera et commentera proposent un portrait pittoresque de la vie dans les Andes. Mais on ne 
peut pas nier, non plus, la poésie qui se dégage des images lorsqu'il filme les paysages de la Cordillère ou la côte pacifique, qui rappelle sans aucun doute les descriptions romantiques de certains explorateurs européens du XIx siècle. Ainsi, Terray offre au spectateur des images qui correspondent avant tout à ce qu'il est et ce qu'il est venu chercher: un explorateur curieux en quête d'aventures venu observer la vie des Indiens.

6 Les plans d'ensemble l'emportent largement dans la construction de ces images. Terray opte très rarement pour des plans rapprochés ou serrés, préférant les plans larges ou moyens qui lui permettent d'offrir une vision globale d'un groupe ou d'un lieu animé, qu'il balaye parfois de sa caméra dans un panoramique horizontal. Cette manière de filmer montre à quel point il veut "embrasser" largement les scènes auxquelles il assiste, comme s'il voulait ne rien rater et rapporter le plus de choses. D'un autre côté, on voit clairement que ce qui l'intéresse n'est pas l'homme indien en tant qu'individu, mais les hommes indiens ou plutôt la culture indienne. Ceci montre que Terray conçoit les Péruviens autochtones comme appartenant à une catégorie ethnique homogène, ne distinguant ni les identités individuelles ni la diversité des communautés. Il filme sans distinction les Quechuas de Ollantaytambo, près de Cuzco, et les Aymaras de Puno, dans l'Altiplano, les englobant dans une seule et même catégorie: les Indiens quechuas. Ces populations ne partagent pourtant ni la même langue ni la même origine ethnique.

7 En prenant soin de ne jamais apparaître sur la pellicule lorsqu'il filme les Péruviens, Terray s'inscrit ouvertement dans une démarche testimoniale. Il semble tenir à ce que ses vidéos se rapprochent le plus possible d'une observation, d'une collecte d'images, comme le proposent les documents ethnographiques des chercheurs auxquels il se réfère parfois (Alfred Métraux, par exemple) ou les documentaires qui font leur apparition à la télévision française dans les années 1950, placés sous l'égide d'explorateurs bien connus, tels que Paul Rivet ${ }^{4}$. Les images de Terray, bien qu'enregistrées en $16 \mathrm{~mm}$ sans son, présentent parfois un accompagnement sonore lorsqu'elles sont montées : musique ou commentaires, voire les deux. Dans le premier cas, Terray choisit d'apposer de la musique traditionnelle andine systématiquement sur la bande (guitare, flûte de pan, percussions), ce qui a probablement pour objet de donner davantage d'authenticité encore, même si on perçoit parfois, dans les séquences de danses et de défilés rituels, que le rythme de la musique, captée lors d'une autre fête, n'est pas raccord avec les images. On ne sait d'ailleurs pas exactement quelle est cette musique ni d'où elle est tirée, Terray se contentant d'indiquer, dans le bandeau d'introduction, «Musique du folklore péruvien ». Les commentaires, quant à eux, sont destinés à expliciter le contenu des images pourtant déjà très explicites. En dehors de cette redondance assez caractéristique des documentaires de l'époque, les explications, proposées par le cinéaste lui-même, décrivent ici les costumes traditionnels, là les techniques de battage de l'orge ou encore les particularités des corridas andines. Dans son exposé, il livre également au spectateur ses sentiments sur la vie des Indiens quechuas et, tout comme les propos qu'il tient dans son autobiographie, ses remarques laissent transparaître une représentation des Indiens assez ambivalente.

8 La manière dont il parle de la vie de ces gens laisse percevoir, d'emblée, sa curiosité pour l'Autre (sans doute un héritage familial que ses expéditions autour du monde n'ont fait que renforcer). En s'appliquant à décrire «[...] la couleur et l'originalité des vêtements, la bizarrerie des coutumes [qui] provoquent parfois un dépaysement plus 
grand encore que celui qu'on éprouve au cœur de l'Asie » (Terray, 1961 : 446), il montre qu'il a conscience de la diversité culturelle. Il reconnaît l'Autre dans sa différence et c'est précisément ce qui le pousse dans sa démarche cinématographique : il se plaît à faire découvrir une autre façon de vivre. Les termes qu'il emploie traduisent son emballement pour ces terres et ses gens; il se dit «[...] littéralement envoûté par le charme sauvage qui se dégage de ce peuple d'Indiens" qu'il décrit comme « magnifiques et colorés » ou encore « hospitaliers et aimables» (ibid. : 424). Dans ses films, il ne tarit pas d'éloges, non plus, sur les savoirs transmis depuis l'époque incaïque, «la précision admirable » avec laquelle les blocs de pierre du Machu Picchu ont été taillés et encastrés, et "les siècles de patience et d'expérience ", dit-il, qui ont permis de domestiquer la vigogne (film C'est le Pérou). On repère néanmoins dans le discours de Terray quelques adjectifs qui contrastent avec cette vision exaltée : «les pauvres indiens », «le misérable peuple des Andes », «les misérables Indiens » (film C'est le Pérou et Terray, 1961:423). S'ils traduisent une forme d'apitoiement sur le sort de ces gens lorsqu'il décrit la situation d'asservissement que connaissent les Indiens des haciendas ou le dénuement dans lequel ils vivent, ces termes évoquent également un certain mépris, notamment lorsqu'ils sont assortis d'autres formules infériorisantes: «[...] nous étions partis à trois dans un massif sauvage à peine peuplé par quelques misérables Indiens que quatre siècles de conquête ont ramenés à l'état de bêtes farouches. » (Terray, 1961: 423) D'autres descriptions font échos à ces considérations typiquement ethnocentrées, certaines juste suggérées, d'autres non dissimulées : ainsi apprend-on que les Indiens sont "malpropres et grossiers ", "voleurs et brutaux", " sournois et ivrognes " (ibid. : 424), " qu'ils ont renouvelé l'exploit de la préhistoire " en poursuivant l'élevage des camélidés, ou encore que "c'est au treizième siècle - [alors que] chez nous, Notre Dame de Paris était déjà bâtie - que leur petite tribu montagnarde fonda [...] Cusco » (film C'est le Pérou). Terray dépeint donc les Indiens également comme un peuple de sauvages, primitifs, arriérés, ce qui n'est pas sans rappeler certains discours chez les chroniqueurs de l'époque coloniale. Ainsi, derrière cette caméra qui cherche à proposer un témoignage authentique et qui commence à considérer l'Autre et essayer de le comprendre, incarnant la mutation de la pensée qui est en train de s'opérer dans les années 1950, se cache en réalité un regard qui ne parvient pas encore à s'affranchir de l'héritage de la pensée impérialiste européenne.

\section{Représentations des Amérindiens en France}

Concernant les images ayant précédé celles tournées par Terray, l'historique des portraits des Indiens du Pérou proposés par des Français fait remonter les premières descriptions au XVII ${ }^{e}$ siècle, après que la Couronne espagnole ait autorisé l'Académie royale des sciences française à organiser des expéditions scientifiques dans ses colonies américaines qu'elle contrôle jalousement. Mais c'est avant tout la nature latinoaméricaine qui intéresse les naturalistes (faune, flore, climat, minéraux, etc.). Il faudra attendre le $\mathrm{XIX}^{\mathrm{e}}$ siècle, notamment sa seconde moitié avec le développement de l'archéologie, pour voir fleurir dans les rapports et carnets de voyage des explorateurs français des passages entiers consacrés aux Indiens des Andes. La naissance de l'égyptologie a fait naître un véritable engouement pour les " antiquités », poussant les collectionneurs-archéologues amateurs à prendre le chemin du Pérou en quête d'objets anciens. Dans leurs notes, ils détaillent la progression de leur voyage, la découverte des sites préhispaniques et livrent leurs impressions sur la vie dans les Andes. Ainsi, 
Laurent de Saint Cricq, membre de l'expédition menée par Castelnau dans les années 1840, décrit les différences qu'il observe entre l'Indien de la côte et celui des montagnes: le premier a "le masque rond, le nez aplati, les lèvres lippues, les yeux étroits à sclérotique jaune, obliques et bridés par les coins comme ceux des Chinois et des races mongoles", tandis que l'autre possède un "facies ovale, des pommettes saillantes, un nez en bec d'aigle, des yeux obliques mais bien fendus, une chevelure noire, abondante et lisse, [et] semble rattaché à la grande famille indoue [...]». Il termine son observation en indiquant que « Du mélange des deux races de la côte et de la sierra, il est résulté avec le temps bon nombre d'hybrides, dont une laideur hébétée est le trait distinctif » (Marcoy $\left.{ }^{5}, 1869: 60\right)$. Ces observations raciologiques, récurrentes dans les journaux des voyageurs qui découvrent le Pérou et ses habitants à cette période, s'accompagnent parfois même de théories originales qui affirment qu'en grandissant, il arrive aux enfants indiens, " comme aux races inférieures telles que le chimpanzé, l'orang-outan et le gorille, que la partie inférieure du crâne et les mâchoires s'avancent à la recherche de la pâtée ", comme l'indique l'archéologue Théodore de Ber dans les années 1870 (Riviale \& Galinon, 2014 : 408). En considérant les Indiens comme une catégorie inférieure d'humains, sortes d'infrahommes, les voyageurs européens ne font en réalité que reprendre les théories anthropologiques de l'époque qui s'emploient à distinguer et hiérarchiser les races. Mais le discours sur les Indiens évolue aussi : on les décrit toujours comme des hommes sales et primitifs, mais on considère aussi leur paresse et leur apathie comme le résultat d'une colonisation mal menée qui a conduit le Pérou à sa perte.

Courbé sous la lame d'acier, l'Indien apprenait à se prosterner à genoux devant la croix espagnole. Tremblant de peur, ahuri, indifférent, répétant sans les comprendre les prières adressées à un Être qu'il ne connaissait point, l'Indien semble avoir désappris le travail en écoutant la messe. Les Indiens avaient été d'excellents agriculteurs, on en a fait des mauvais mineurs; aller à la mine c'était aller à la mort. C'est ainsi que l'activité s'est éteinte, et que la nature des Andes au caractère inhospitalier a reparu pendant que les vastes cultures dont l'Indien l'avait recouverte se rétrécissaient de plus en plus. (Wiener, 2010 [1880] : 303)

Vers la fin du XIX ${ }^{e}$ siècle, au moment où l'ethnologie se développe, on craint désormais de voir disparaître les «races pures » et leurs mœurs sous l'effet du processus de métissage. L'invention de la photographie permet de figer les traits de ces gens ; ainsi, en 1889, la Société d'anthropologie de Paris invite les explorateurs à réaliser des clichés des populations qu'ils rencontrent: "Il faut réunir les portraits des hommes, des femmes, des enfants, des vieillards, des adultes surtout, avec les costumes, [...]. Il faut se hâter car l'unification marche à grands pas.» $(1889: 303)$ Bien souvent, les portraits "ethniques» se rapprochent davantage des «natures mortes», les Indiens posant devant une toile unie dans des positions parfois peu naturelles. Cette chosification en est d'autant plus accentuée lorsque les titres des photos réduisent les modèles à des catégories de métiers ou à des lieux, leur refusant ainsi toute identité individuelle, comme les photos que fit l'archéologue Charles Wiener en $1875^{6}$. Une trentaine d'années plus tard, les époux d'Harcourt sillonnent le Pérou pour enregistrer la musique traditionnelle des Indiens quechuas ; ils craignent « l'effacement de l'élément indigène en faveur d'une musique affreusement mélangée " (Harcourt, 1959:6), abâtardie sous l'influence de la musique occidentale apportée par l'arrivée du phonographe, de la radio et du flot de touristes. Les photos que ramèneront les d'Harcourt de leurs terrains de 1913 et 1919 contrastent avec celles de la fin du siècle passé. Ce ne sont plus des portraits, mais des vues d'ensemble d'une place ou d'une rue, 
où se trouvent des gens; les photos sont prises sur le vif, sans préparation, capturant un moment de vie ${ }^{7}$. Les travaux des d'Harcourt et d'autres ethnologues s'intéressent désormais non plus aux Indiens, mais à leur quotidien et leurs pratiques culturelles : musique, savoirs techniques, langues, etc. Ce déplacement des objets d'étude illustre l'évolution du regard scientifique qui reconnaît maintenant la diversité culturelle chez les autochtones des Andes. Mais si on étudie les caractéristiques des langues ou des vêtements de telle ou telle communauté, c'est pour consigner leurs spécificités avant que ce patrimoine immatériel ne disparaisse. Car c'est dans l'ordre des choses, l'unification de la population est la prochaine étape de l'histoire latino-américaine et il faut encourager l'assimilation "des races autochtones ", comme le dit Paul Rivet. Ce spécialiste des langues subamérindiennes, directeur du Musée de l'Homme de 1937 à 1949, écrit en 1948 un article intitulé « Sur l'Amérique latine : propos d'un ami », dans lequel il livre ses espoirs pour la résolution du « problème indien » en Amérique latine. Il indiquera que pour ce faire, «Il s'agit de chercher les meilleures méthodes pour éduquer ces masses allogènes, leur donner, peu à peu, une culture d'inspiration blanche et les incorporer à la nation » $(1948: 395)$. Au début des années 1950 naît le Programme indien-andin (1953-1974) : un plan d'assistance technique créé par l'Agence des Nations unies, qui a pour but d'accompagner les Indiens dans leur "développement» économique, social et culturel (programmes linguistiques, professionnels, éducatifs, etc.). Des anthropologues sont intégrés au programme pour apporter leur regard d'experts sur la faisabilité et la mise en place de ces projets. Alfred Métraux participe à une mission en 1954 au Pérou, puis en Bolivie 2 ans plus tard. Dans un article publié dans la revue Le Courrier de l'UNESCO, il explique que « l'amélioration de leur niveau de vie est inconcevable sans un renouvellement de presque tous les aspects de leur culture hybride" (1955:6), n'hésitant pas à reprendre des images stéréotypées qui les dépeignent comme des êtres arriérés végétant dans leur saleté ${ }^{8}$.

Les documents audiovisuels français consacrés au Pérou et les films qui précèdent ceux tournés par Lionel Terray sont particulièrement rares. Une recherche dans les archives du Centre national du cinéma et de l'image animée et dans celles de l'Institut national de l'audiovisuel montre qu'avant 1952, seuls un film et un documentaire ont été diffusés en France : un document muet de 1931 au sujet de la récolte du guano au Pérou, commandé par le ministère de l'Agriculture, et la version française d'un reportage allemand sur l'histoire préhispanique du Pérou, diffusé en $1950^{\circ}$. Le premier n'offre que de rares plans sur les travailleurs péruviens ramassant l'engrais naturel. Le second s'attarde largement sur l'héritage inca dans les pratiques culturelles indiennes; on note dans les commentaires un manque d'aisance dans la manipulation des termes autochtones (Titicac) et quelques mélanges et approximations (notamment l'amalgame avec les cultures préhispaniques maya et aztèque qui n'appartiennent ni à la même aire géographique ni à la même période). Ceci montre à quel point le Pérou est loin de l'Hexagone et méconnu. La démocratisation de la télévision dans les foyers français dans les années 1950 et le développement des expéditions anthropologiques vont favoriser l'émergence d'émissions dédiées à la découverte de la vie dans des contrées lointaines, telles que Voyage sans passeport, qui diffuse 156 reportages entre 1956 et 1969 ; parmi eux, cinq numéros sont consacrés au Pérou entre 1959 et 1960. Trois d'entre eux sont des images tournées par Lionel Terray: il s'agit en réalité de son film muet Hommes et cimes du Pérou qui a été divisé en trois parties et adapté au format de la télévision ${ }^{10}$. Les commentaires de la voix off sont ceux des réalisateurs de l'émission et apportent un éclairage sur le regard que la société 
française portait sur les Indiens péruviens à cette époque. Les Indiens y sont présentés comme des gens "honnêtes, travailleurs et hospitaliers "; on décrit la splendeur des tissus qu'ils réalisent grâce aux techniques héritées de leurs ancêtres incas et on applaudit leur agilité pour attraper le bétail. Mais les remarques positives sont assez rares et le discours est presque unilatéral ; il insiste avant tout sur le caractère primitif des Indiens et la vie de débauche qu'ils mènent: ce sont des "misérables" vêtus de « loques » et qui vivent dans des « huttes plus que sordides" ou des "maisons en pisé parfois délabrées »; la forte mortalité infantile est due à « l'usage excessif d'alcool, [...] l'effet toxique de la coca et aussi un total manque d'hygiène " indique-t-on. La conclusion est sans appel : pour remédier à la « décadence de la race indienne [...] il suffirait de peu de choses [...], probablement un léger travail d'éducation ». L'image qui est donnée des autochtones est encore fortement influencée par le sentiment de supériorité européen qui a fait du progrès le critère fondamental d'évaluation des sociétés, ce technocentrisme qui a servi les desseins coloniaux et plus tard les politiques interventionnistes comme le Programme indien-andin par exemple.

\section{Considération pour les Amérindiens au Pérou}

12 À cette époque, au Pérou, les populations des montagnes andines constituent une masse encore fortement marginalisée, malgré les réformes, les décrets, les lois promulguées pour gommer la fracture sociale entre Indiens et non-Indiens et " améliorer » leurs conditions de vie. Au XvI ${ }^{\mathrm{e}}$ siècle, déjà, des voix se sont élevées pour dénoncer l'usurpation des droits des Indiens et leur exploitation, comme le fit Bartolomé de las Casas, entraînant la création des Nouvelles Lois qui n'ont cependant pas fait que profiter aux Indiens (la suppression de la perpétuité des encomiendas, puis la suppression complète des encomiendas, en 1542, a permis à la Couronne de récupérer la pleine possession de ces terres et des Indiens qui y vivaient). Mais par la suite, et tout au long de la période coloniale, les différentes révoltes indiennes se sont systématiquement soldées par de violentes répressions et des exécutions magistrales (décapitation, écartèlement sur la place publique, dont l'exemple le plus connu est certainement celui de Túpac Amaru exécuté publiquement en 1572). Plus tard, au moment des indépendances latino-américaines, on a clamé l'égalité des Blancs et des Indiens et construit les jeunes républiques à partir de ce critère. En 1815, Simón Bolívar, protagoniste clé de la guerre des indépendances latino-américaines, posait pourtant un regard générique, réducteur et méprisant sur «l'indien», le décrivant comme un bon sauvage doux, pacifique, indolent et sans ambition :

L'Indien est de caractère paisible; il ne souhaite que le repos et la solitude: il n'aspire pas à commander sa tribu, encore moins celle des autres [...]. Il ne réclame aucune autorité, car il n'en a pas l'ambition ni ne pense être apte à l'exercer, se contentant de sa tranquillité, sa terre et sa famille ${ }^{11}$.

13 L'indépendance du Pérou acquise (1821), la toute nouvelle république, consciente de la bipolarisation de la société, a cherché à intégrer officiellement les Indiens et a déployé un arsenal de mesures et de lois en ce sens. Inscrit dans une démarche de construction nationale, l'État place ces mesures dans un projet d'homogénéisation de la nation - projet clairement assimilationniste. En 1825, plusieurs décrets en faveur des Indiens sont promulgués par Bolívar à Cusco, dont l'interdiction du service personnel aux créoles (réduisant l'exploitation tacite des Indiens) et la répartition des biens des communautés indiennes en lots (censée développer la production agraire 
sous l'effet de la propriété privée). Paradoxalement, Bolívar s'oppose aussi farouchement à l'intégration raciale des Indiens dans la société, c'est-à-dire au métissage : cela signifie, pour lui, la mise en marche d'une abâtardisation des races et la dégénérescence de la population américaine (Favre, 1986:14) ${ }^{12}$. De son côté, José de San Martín, dans un décret qu'il publie au lendemain de la déclaration d'indépendance du Pérou, annonce que « Désormais les autochtones ne seront plus appelés Indiens ou naturels; en tant qu'enfants et citoyens du Pérou, ils doivent être connus sous le nom de Péruviens ${ }^{13} »$. Cet ajustement terminologique est hautement symbolique et illustre à quel point on cherche à faire disparaître la composante ethnique indienne de la réalité sociale péruvienne en "l'absorbant». Si les Indiens sont bien des citoyens péruviens, leur condition sociale influera cependant directement sur certains de leurs droits: au fil des constitutions, les Péruviens autorisés à voter devront, par exemple et selon les époques, être propriétaires, ou bien savoir lire et écrire, écartant de ce fait une grande majorité d'Indiens ${ }^{14}$.

14 Le début $\mathrm{du} \mathrm{xx}^{\mathrm{e}}$ siècle constitue une période charnière; il marque le début de "l'indigénisme», ce mouvement social, politique et artistique qui plaide la cause des masses indiennes opprimées et réfléchit à des mesures en leur faveur. L'essayiste Manuel González Prada publie dès 1904 Nuestros Indios dans lequel il condamne le darwinisme social qui soutient que les races ont des capacités inégales et que «l'Indien » est « réfractaire à la civilisation » $(1904: 14)^{15}$. Dans son essai, il juge que seuls les Indiens ont le pouvoir de mettre fin à l'oppression qui les maintient asservis depuis la conquête; il les appelle à se soulever sans attendre, leur suggérant notamment d'investir dans des armes et des balles plutôt que de gaspiller leur argent dans l'alcool et les fêtes et d'attendre que le cœur des Blancs s'attendrisse sur leur sort (ibid.:19). En plus de l'image générique et stéréotypée - «l'Indien» ivrogne et apathique -, on remarque également dans son discours l'expression "le problème indien » (qui sera reprise par d'autres plus tard: Mariátegui, 1928), formulation qui laisse envisager que "l'Indien » est le problème et non que la société a un problème avec les Indiens.

15 Avec la nouvelle Constitution de 1920, le gouvernement péruvien, inscrit dans une dynamique de modernisation du pays, propose des mesures qui octroient des droits spéciaux aux communautés indiennes: reconnaissance de la diversité ethnique et culturelle, reconnaissance de l'existence juridique des communautés indiennes et imprescriptibilité de ce statut ${ }^{16}$. Cependant, la politique indigéniste du président Augusto Bernardino Leguía répond avant tout au projet national de modernisation du pays. S'il se considère comme un défenseur de « la race indienne » et qu'il soutient les actions des organisations pro-Indiens indépendantes (engagées dans la défense des Indiens au tribunal ou dans des campagnes d'alphabétisation), il cherche rapidement à les contrôler pour orienter les programmes en faveur des Indiens ${ }^{17}$. L'objectif prioritaire est de favoriser le « développement» des communautés pour faciliter leur intégration dans la société péruvienne. L'État promulgue par ailleurs, en 1924, un nouveau code pénal (connu comme Código Penal Maúrtua) qui propose des peines spéciales pour les Indiens "sauvages [...] semi-civilisés ou abrutis par l'esclavage et l'alcoolisme ${ }^{18}$ ». Ce code pénal restera en vigueur jusqu'en 1991. La Constitution de 1933 apporte quelques changements notables, particulièrement concernant les biens fonciers des Indiens (l'État s'engagera par exemple à fournir des terres aux plus 
démunis et à garantir leur inaliénabilité), mais continue à maintenir l'alphabétisation comme condition au droit de vote.

En 1946, sous l'impulsion de la création de l'Institut indigéniste interaméricain (1940) où sont discutées les politiques indigénistes de chaque pays, naît l'Institut indigéniste péruvien. Organe du ministère du Travail qui se maintiendra jusqu'en 1981, il a pour but de réfléchir à la création de nouvelles mesures, de faire appliquer les lois et de coordonner les projets. Certains lui reprocheront, plus tard, d'avoir élaboré des programmes destinés à lisser l'image de l'Indien pour qu'il s'insère dans l'idée de nation qui était en train d'être mûrie : des programmes éducatifs qui reposaient sur la castellanisation et des programmes de santé réduits à une sensibilisation à l'hygiène et, parfois, une protection des traditions anciennes pour que soit exposé dans les musées ce qui fait l'orgueil du pays (Gonzáles, 2011 : 133-158).

Pendant el Ochenio de Odría (dictature de 1948 à 1956), on continue de réfléchir aux manières de résoudre «le problème indien » dans une démarche assimilationniste. Les programmes s'inscrivent désormais dans une perspective scientifique et les Indiens deviennent objet d'étude. L'État péruvien signe, par exemple, un accord avec l'Université nord-américaine de Cornell qui mène un projet anthropologique expérimental dans la région de Ancash, destiné à moderniser les Indiens qui vivent à Vicos. Là-bas, une hacienda est transformée en terrain d'étude et d'application: on observe les résultats d'apprentissage de quelque 2000 Indiens à qui on enseigne les modèles de la vie moderne (notamment la technologie agraire). Dans un long article intitulé "L'anthropologie culturelle et "notre problème indien": Vicos, un cas d'anthropologie appliquée ", l'un des anthropologues en charge du projet, le Péruvien Mario Vázquez Várela (1952), indiquait à l'époque que la société péruvienne reposait sur un socle culturel binaire avec, d'un côté, des modèles de culture occidentale et, de l'autre, des modèles de peuples arriérés. Ceci laisse entrevoir le regard que le gouvernement péruvien pose sur la population indienne au moment où Lionel Terray tourne ses images.

Les représentations des Indiens proposées par la société péruvienne de cette première moitié $\mathrm{du} \mathrm{xx}$ siècle, notamment dans la photographie et les films, illustrent, quant à elles, l'autre versant du mouvement indigéniste. Car, si avant les années 1950, les Indiens ne sont pas représentés dans le cinéma péruvien ${ }^{19}$ - illustrant bien la négation de leur existence -, le Club de Cusco, à l'inverse, fera de l'identité indienne son sujet de prédilection dès 1955. Bien que l'industrie du cinéma au Pérou ne parvienne pas à se développer, écrasée sous le poids des productions nord-américaines ou mexicaines et abandonnée par le gouvernement péruvien, un groupe de cinéastes indiens s'engage dans la célébration de la culture indienne et la dénonciation de sa marginalisation, comme d'autres le feront en littérature ${ }^{20}$. Ainsi, plusieurs courts ou longs métrages autofinancés (et donc éloignés des modèles que veut imposer le gouvernement) sortent dans les années 1950 : Corpus en la ciudad de Cusco (1955), Corrida de toros y cóndores (1956) de Manuel Chambi (en coréalisation avec Eulogio Nishiyama) ou encore Policromía andina (1958) de Luis Figueroa Yábar. Tournés comme des documentaires, ils s'inscrivent dans une volonté d'intégrer l'identité indienne dans le cinéma péruvien et proposent des images éloignées des stéréotypes présents dans les documents occidentaux. Ils se situent par ailleurs dans la lignée des premiers photographes péruviens indigénistes, Martín Chambí (originaire de l'Altiplano) et Daniel Cisneros Cáceres (natif de la région de Cusco) qui ont accordé une large place aux Indiens dans 
leurs travaux. Pendant les premières décennies du $\mathrm{xx}^{\mathrm{e}}$ siècle, les clichés saisis au Pérou ont fait la part belle aux portraits de studios, pensés comme des tableaux figés, commandés par des clients argentés (en grande majorité créoles ou métisses); et lorsque les photographes sortaient de leur atelier, ils choisissaient d'immortaliser des sujets statiques qui ne risquent pas de compromettre la netteté de la photo (vues panoramiques de grandes villes, monuments, montagnes) ${ }^{21}$. La photographie de "plein air ", qui se développe en même temps que l'évolution des techniques, a ouvert de nouvelles perspectives : les photographes se sont alors intéressés aux scènes de vie et aux différentes classes socioprofessionnelles dans leurs activités (militaires, mineurs, religieux, écoliers, ouvriers ${ }^{22}$. La diversification des thèmes photographiés, à un moment où fleurissent les arts indigénistes qui mettent en valeur l'identité autochtone, permet progressivement aux Indiens de figurer parmi les sujets d'intérêt pour certains photographes, surtout ceux d'origine amérindienne. C'est ainsi que Martín Chambi et Daniel Cisneros Cáceres, après avoir appris la photographie à Arequipa, retournent dans les hautes terres pour documenter la vie des hommes et des femmes des Andes, leurs fêtes et leurs coutumes. Martín Chambi deviendra l'un des photographes péruviens les plus reconnus internationalement. Ses photos, qui oscillent entre témoignage documentaire et esthétisme, dépeignent la vie dans les Andes et font écho à l'identité du photographe. À sa mort, il laisse plus de 30000 négatifs; on lui consacrera des expositions dans différents pays d'Amérique latine, en Europe et aux États-Unis.

\section{Conclusion}

Les films-portraits des Indiens du Pérou que propose Lionel Terray correspondent au regard que la France ou l'Occident porte sur les populations des montagnes andines à la même époque. Ils donnent à voir plusieurs images de "l'Indien", parfois très réductrices et stéréotypées (comme l'illustre l'emploi de l'article défini singulier et générique, qui rejette les identités plurielles indiennes), d'autres fois romantiques ou encore pittoresques. Elles reflètent la mutation du regard de la société française, à cheval entre ethnocentrisme hérité de la pensée coloniale, curiosité de touriste et reconnaissance de l'Autre. Elles traduisent également la personnalité plurifacétique de Terray: un explorateur conquérant, mais aussi un aventurier curieux. On repère toutefois une évolution dans les images qu'il ramène du Pérou : les premières insistent davantage sur l'aspect rudimentaire de la vie des Indiens, la misère et leurs déviances, offrant ainsi l'image d'une population arriérée; les dernières montrent plutôt les savoirs et traditions hérités de la période préhispanique, montrant la vivacité des traditions et la force de l'identité indienne. L'une comme l'autre concordent avec l'image que le gouvernement péruvien de l'époque utilise pour assimiler cette population exclue : des Indiens qu'il faut moderniser, mais dont le folklore doit être préservé. 


\section{BIBLIOGRAPHIE}

BOLíVAR Simón (2014), Reflexiones políticas, Barcelone : Linkgua Digital.

FAVRE Henri (1986), « Bolívar y los indios », Historica, 10(1), 1-18.

GoNZÁLEZ Osmar (2011), « El Instituto Indigenista Peruano: una historia por conocer », L. Giraudo \& J. Martín Sánchez (dir.), La ambivalencia del Indigenismo. Campo Interamericano y trayectorias nacionales 1940-1970, Lima : IEP, 133-158.

GonZÁlez PRADA Manuel (1905), « Nuestros indios », Latinoamerica, Cuadernos de cultura

latinoamericana, 29.

HARCOURT Raoul \& Marguerite d' (1959), « La musique des Aymaras sur les hauts plateaux boliviens », Journal de la Société des américanistes, 48, 5-133.

MARcoy Paul (1869), Voyage à travers l'Amérique du Sud, de l'Océan Pacifique à l'Océan Atlantique par Paul Marcoy (vol. 1), Paris : Hachette.

MÉTrauX Alfred (1955), «Les indiens des Andes souffrent... de "la faim de la terre" », Le Courrier de l'UNESCO, 8(2), 4-9.

SociÉTÉ D'ANTHRopologie De PARIS (1889), Photographie ethnique, Participation à l'Exposition universelle de 1889 de la Société d'anthropologie de Paris, Paris : Imprimerie nationale.

Rivet Paul (1948), «Sur l'Amérique latine : propos d'un ami », Annales, Économies, Sociétés, Civilisations, 3(4), 393-400.

Riviale Pascal \& GALINon Christophe (2014), Une vie dans les Andes : le journal de Théodore Ber (1864-1896), Paris : Ginkgo.

TERRAY Lionel (1961), Les conquérants de l'inutile, Paris : Gallimard.

VARELA VÁzquez Mario (1952), « La antropología cultural y nuestro problema indio: un caso de antropología aplicada », Perú Indígena, 2(5-6), 7-157.

WiENER Charles (2010), Voyage au Pérou et en Bolivie (1875-1877) [1880], Paris : Ginkgo.

\section{Sitographie (Fonds d'archives)}

Films de Lionel Terray : CIMALPES (Cinémathèque d'images de Montagne), <www.cimalpes.fr> (30 juin 2020).

Photographies de Charles Wiener et des époux d'Harcourt : Musée du Quai Branly, $<$ www.quaibranly.fr> (30 juin 2020).

Reportages et émissions sur le Pérou : INA (Institut national de l'audiovisuel), <http://ina.fr> (30 juin 2020).

\section{NOTES}

1. Formule qu'il choisit pour décrire sa famille dans son autobiographie Les conquérants de l'inutile, publiée en 1961 (Terray, $1961: 4$ ). 
2. Marcel Ichac a joué un rôle majeur dans le domaine technique de la réalisation de films d'exploration en optant pour le $16 \mathrm{~mm}$ dont les caméras offraient le double avantage d'alléger le poids du matériel et un tournage en couleurs qui ne soit pas onéreux.

3. Victoire sur le Huantsán (1952, 26'25) ; Le monde du vertige (21'09) ; Fête quechua (01'37 et 02'01) ; Hommes et cimes du Pérou : Soray (26'36); Hommes et cimes du Pérou : Cusco, Arequipa, Lima (34'46); Hommes et cimes du Pérou: Deuxième partie, Cusco (09'14); De Ande expeditie (1956, 16'41); C'est le Pérou (1964, 14'44). Archives du CIMALPES.

4. Le premier numéro du Magazine des explorateurs a été présenté par Paul Rivet le 23 février 1956.

5. Paul Marcoy est le pseudonyme sous lequel a écrit Laurent de Saint Cricq.

6. Charles Wiener est parti en mission archéologique entre 1875 et 1877. Musée du Quai Branly, Fond photographique, PP0024397 à PP0024412.

7. Musée du Quai Branly, Fond photographique PP0010186, PP0010189, PV0010189, PV0036341.

8. «Ce n'est pas sans raison qu'ils ont la réputation d'être parmi les peuples les plus sales de la terre! L'inventaire des maladies dont ils sont atteints a de quoi faire frémir. »

9. Le guano naturel du Pérou (1931). Au pays des Incas (1950). Archives de l'INA.

10. "Pérou : Cuzco et Lima ", "Pérou : les Andes » (1959). "Pérou ", "Pérou: l'Altiplano » et " Pérou : le lac Titicaca » (1960). Numéros de l'émission Voyage sans passeport. Archives de l'INA.

11. Lettre du 28 septembre 1815 faisant partie de la série d'articles qu'il publia comme des lettres dans The Royal Gazette de Kingston. Nous traduisons.

12. L'auteur prend appui sur plusieurs lettres. Bolívar est convaincu que la population refusera, de toute façon, de se mélanger aux Blancs qui les ont dominés pendant des centaines d'années.

13. Décret du 27 août 1821. Nous traduisons.

14. Constitution de 1823 (article 17), Constitution de 1826 (article 14). La loi électorale de 1890 écarte les analphabètes des élections.

15. Nous traduisons.

16. Article 58 de la Constitution de 1920.

17. Il remplacera par exemple en 1924 le Comité Pro-Derecho Indígena Tawantinsuyu, indépendant, par le Patronato de la Raza Indígena, dépendant de l'État.

18. Article 44 et 45 du code pénal de 1924.

19. Les rares films de l'époque sont soit des documentaires sur la vie galante de Lima, soit des fictions montrant des problématiques typiquement créoles ou encore des documents utilisés comme instrument par la Escuela Cinemática Ambulante pour l'éducation massive des Indiens et leur intégration au processus national.

20. José María Arguedas, Yawar Fiesta (Fiesta de Sangre, 1941), ou Uku Mayu, Ríos profundos (1958).

21. Les photos de Max T. Vargas, par exemple.

22. Les frères Carlos et Miguel Zargas, par exemple.

\section{RÉSUMÉS}

Grande figure de l'alpinisme français de l'après-guerre, membre de huit expéditions, notamment dans les Andes et l'Himalaya, spécialiste des premières ascensions, Lionel Terray était passionné par la montagne, le cinéma et "poussé par la curiosité des hommes", selon ses propres mots (C'est le Pérou, film qu'il réalise en 1964 en collaboration avec Marcel Ichac et Jean-Jacques Languepin). Lors des ascensions de plusieurs sommets enneigés au Pérou en 1964 et à l'occasion 
de séjours postérieurs, il réalise des images sur la vie et les traditions des Indiens du Pérou; il rapporte en France quelques bobines de film à partir desquelles il réalisera différents documentaires, dont l'un gagnera le Grand Prix du Film de montagne et de l'exploration au Festival international de Trente.

Les films-portraits des Amérindiens du Pérou que propose Lionel Terray donnent à voir plusieurs images de "l'Indien", parfois très réductrices et stéréotypées, d'autres fois romantiques ou encore pittoresques. S'ils reflètent la personnalité de leur auteur - un explorateur conquérant, mais aussi un aventurier curieux -, ils traduisent également la mutation du regard de la société française à l'égard des habitants des contrées lointaines, à cheval entre ethnocentrisme hérité de la pensée coloniale et reconnaissance de l'Autre, à une époque où la nation péruvienne peine à admettre sa pluralité culturelle.

Lionel Terray is a great figure of post-war French mountaineering. He took part into eight expeditions, particularly in the Andes and the Himalayas, and was a specialist of first ascents. Lionel Terray was a passionate lover of mountains, of cinema and was "driven by his curiosity for mankind". While climbing several snow-capped peaks in Peru in 1956 and during later stays, he shot footage of the life and traditions of Indigenous people of Peru. He brought with him a few reels back to France, from which he made various documentaries, one of which won the Grand Prix of Mountain Film and Exploration at the Trento International Film Festival.

Lionel Terray's portraits of Peruvian Indigenous people show several images of "the Indian", sometimes very simplistic and stereotyped, other times romantic or picturesque. If they reflect the personality of their author-a conquering explorer and a curious adventurer-they are also a testimony of the shift in the way the French society looked at the inhabitants of far-off countries, caught between the ethnocentrism inherited from colonial thought and the recognition of the Other, at a time when the Peruvian nation was struggling to acknowledge its cultural plurality.

\section{INDEX}

Mots-clés : représentations, Amérindiens, Pérou, regards, stéréotypes

Keywords : representations, Indigenous people, Peru, look, stereotypes

\section{AUTEUR}

\section{CHLOÉ TESSIER-BRUSETTI}

Université de Nantes (CRINI)

chloetessier@gmail.com 